\title{
Author Correction: TMEM151A variants cause paroxysmal kinesigenic dyskinesia
}

Hong-Fu Li, Yu-Lan Chen, Ling Zhuang, Dian-Fu Chen, Hua-Zhen Ke, Wen-Jiao Luo, Gong-Lu Liu, Sheng-Nan Wu, Wen-Hao Zhou, Zhi-Qi Xiong and Zhi-Ying Wu (D)

Correction to: Cell Discovery (2021)7:83

https://doi.org/10.1038/s41421-021-00322-w

Published online 13 September 2021

In the original publication of this "Correspondence", we made some mistakes in the annotation of genotypes in Fig. 1a.
The correct genotypes in Family 2 are as follows: II1 $(+/+)$, II2 (NA), II3 (+/+), II4 (p.C125X), and II5 $(+/+)$. In addition, a half round bracket was missed after "(p.S297T" in Fig. 1b. The correctly labelled Fig. 1a and b is displayed as below. This correction does not affect the description of the results or the conclusion of this work. a

a

II

III

b

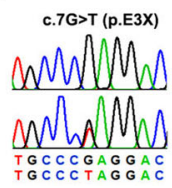

I

II
Family 1
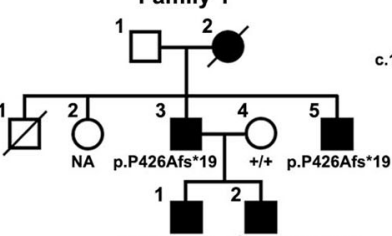

p.P426Afs*19 7 p.P426Afs 19

c.140T>C (p.L47P)

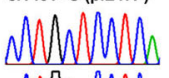

MNAM

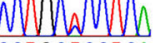

CCTGCTCTCA
CCTGCCCTCA

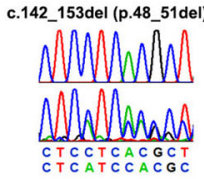

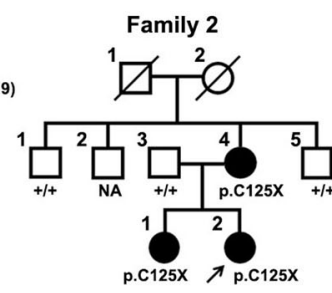

.623_624insA (p.L210Afs) c.739G>T (p.E247X) shwolly JoWManh

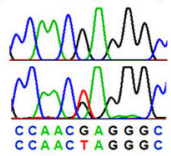

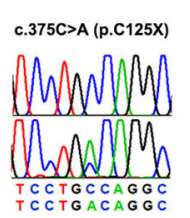

c.863T>C (p.F288S)

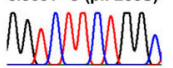

con

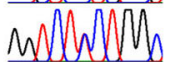

GGTCTTCTGGC
GGTCTCCTGGC

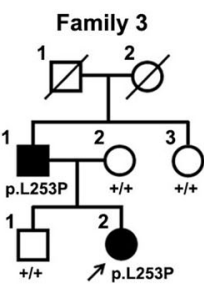

c.758T>C (p.L253P)

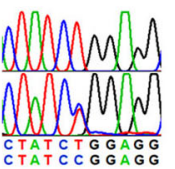

c.889T>A (p.S297T) c.897_912del (p.L300pfs)

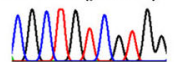

MDMWh

CGCTGTCGTGG
CGCTACGTG

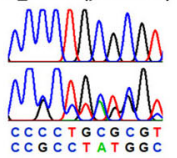

Published online: 28 October 2021

\section{Reference}

1. Li, H. et al. TMEM151A variants cause paroxysmal kinesigenic dyskinesia. Cell Discov. 7, 83 (2021). 